\title{
Identificación genética de recién nacidos en Perú: un estudio piloto
}

\author{
Genetic identification of newborns in Peru: a pilot study
}

\author{
Carlos D. Neyraa ${ }^{\mathrm{a}, \mathrm{b}}$, Marilyn R. Suárez ${ }^{\mathrm{c}}$, Eddie D. Cueva ${ }^{\mathrm{a}}$, Henri Bailon ${ }^{\mathrm{d}}$, Ericson L. Gutiérrez ${ }^{\mathrm{d}, \mathrm{e}}$
}

\author{
aGerencia de Calidad e Innovación, Registro Nacional de Identificación y Estado Civil. Lima, Perú \\ bFacultad de Medicina Humana, Universidad Alas Peruanas. Lima, Perú \\ 'Departamento de Ginecología, Instituto Nacional Materno Perinatal. Lima, Perú \\ ¿Centro Nacional de Salud Pública, Instituto Nacional de Salud. Lima, Perú \\ eFacultad de Medicina Humana, Universidad de San Martin de Porres- Lima, Perú
}

Recibido el 4 de mayo de 2018; aceptado el 30 de septiembre de 2018

\section{Resumen}

Objetivo: Determinar la factibilidad de la identificación genética a un grupo de recién nacidos provenientes de un hospital público de Lima-Perú. Material y Método: Estudio descriptivo de corte transversal, realizado por Registro de Identificación y Estado Civil de Perú, en recién nacidos vivos y sus respectivas madres, provenientes del Hospital Carlos Lanfranco La Hoz (Puente Piedra-Lima) durante el mes de enero del 2015. Las muestras fueron colectadas en tarjetas FTA (Fast Technology for Analysis of nucleic acids) que permitieron un análisis directo por PCR (Polymerase Chain Reaction) y electroforesis capilar de 21 marcadores genéticos de tipo STR (Short Tandem Repeats), incluyendo el marcador amelogenina para la determinación del sexo. Resultados: Se incluyeron un total de 44 madres y 45 recién nacidos (existió un parto gemelar). La probabilidad de maternidad fue mayor al 99.9\% en todos los casos. No se encontraron dificultades en la toma de muestra, ni en el transporte del material. El material biológico obtenido fue suficiente para la obtención de ADN para realizar la identificación del recién nacido. Conclusiones: El procedimiento de identificación genética fue factible de realizar en este hospital. Se identificaron etapas del proceso que podrían mejorarse para la posible aplicación de este procedimiento a una mayor escala en el Perú.

\section{Abstract}

Objective: To determine the feasibility of genetic identification in a group of newborns from a public hospital in Lima, Peru. Material and Method: Descriptive cross-sectional study, carried out by the National Registry of Identification and Civil Status of Peru, on live newborns and their mothers, from the Carlos Lanfranco La Hoz Hospital (Puente Piedra, Lima) during January. 2015. The samples were

\section{Palabras clave:}

Identificación

biométrica;

Identificación genética; Reacción de Polimerasa en Cadena;

$\mathrm{ADN}$;

Perú;

Recién Nacido

\section{Keywords:}

Biometric

identification; genetic identification; Polymerase Chain Reaction; DNA; Peru; Newborn 
collected in FTA (Fast Technology for Analysis of nucleic acids) cards that allowed a direct analysis by PCR (Polymerase Chain Reaction) and capillary electrophoresis of 21 STR markers (Short Tandem Repeats), including the amelogenin marker for gender determination. Results: 44 mothers and 45 newborns were included (there was a twin birth). The probability of maternity was higher than $99.9 \%$ in all cases. There were no difficulties in the sampling or in transporting the material. The obtained biological material was enough to collect DNA to identify the newborn. Conclusions: The genetic identification procedure was possible to perform in this hospital. Stages of the process that could be improved were identified for the eventual application of this procedure on a larger scale in Peru.

\section{Introducción}

La identificación es un derecho inherente del recién nacido, reconocido tanto en el Perú ${ }^{1}$ como a nivel internacional ${ }^{2}$. Actualmente se usa las huellas pelmatoscópicas para la identificación del recién nacido, así mismo a partir de los 8 meses se puede incorporar la huella dactilar, sin embargo estas no garantizan una identificación confiable ${ }^{3}$.

En el Perú, a pesar de los esfuerzos de asegurar la relación madre-hijo a través de la inscripción del nacido vivo y el uso de pulseras de identificación con números idénticos en la madre y el niño en la sala de partos, se han evidenciado casos de vulneración de identidad del recién nacido, causados por errores de cambio, pérdida, rotura, alteración u olvido durante la colocación de dichas pulseras.

En tal sentido, la situación descrita genera ventanas de vulnerabilidad que pueden ser aprovechadas para la trata de niños, para cometer fraudes de identidad contra el grupo vulnerable de los recién nacidos, contra el Estado Peruano y contra la seguridad jurídica del Perú.

El análisis de ADN para la identificación se viene utilizando a nivel mundial frecuentemente en el campo de la criminalística, forense y en la investigación biológica de la paternidad ${ }^{4}$. No obstante, también puede ser utilizada para fines de identificación civil como ocurre en Kuwait ${ }^{5}$.

El objetivo de este estudio es determinar la factibilidad de la identificación genética a un grupo de recién nacidos provenientes de un hospital público de Lima Perú.

\section{Material y Método}

\section{Diseño y población de estudio}

Estudio descriptivo de corte transversal, la población estuvo constituida por recién nacidos vivos y sus madres del Hospital Carlos Lanfranco La Hoz ubicado en el distrito de Puente Piedra-Lima, realizado en el mes de enero de 2015. Al ser un estudio piloto, la selección de los participantes se realizó de forma no probabilística por conveniencia y se trabajó por ello con 45 recién nacidos (incluyendo gemelos) y 44 madres. Los criterios de inclusión de los participantes fueron: recién nacidos de ambos sexos, que realizaron el trámite de Registro de Acta de Nacimiento y/o inscripción del DNI (Documento Nacional de Identidad) por primera vez dentro el hospital; con ambos padres presentes y mayores de edad. Los criterios de exclusión fueron: padres de familia que no hayan deseado participar y madres que hayan tenido trasplante de médula ósea y/o que hayan recibido transfusión sanguínea en los últimos 6 meses, eventos que pudieran eventualmente generar perfiles genéticos del donante.

\section{Herramienta de recolección de datos}

El RENIEC desarrolló el Sistema de Gestión de Identificación Genética en 3 módulos principales: i) Registro de Consentimiento (consentimiento informado firmado); ii) Cargo de envío de muestras biológicas (cadena de custodia) y iii) Registro de Identificación genética; además del registro de responsables.

\section{Toma de muestra}

Se tomó una muestra de sangre del talón del recién nacido por punción con lanceta, y en el caso de la madre se tomó dos gotas de sangre de su dedo índice. Ambas muestras fueron obtenidas en tarjetas FTA separadas (incluye código de barras). Las tarjetas FTA contienen una matriz tratada químicamente que lisa una gran variedad de tejidos (por ejemplo sangre, saliva, entre otros). Luego de lisar las células, el ADN liberado se une a la tarjeta donde la matriz protege a los ácidos nucleicos de agentes dañinos que pudieran producir degradación reduciendo de esta forma la degradación de los mismos ${ }^{6}$.

\section{Obtención del perfil genético}

El análisis de las muestras biológicas las realizó el Laboratorio de Biología Molecular y de Genética (LABIMOG) del Instituto de Medicina Legal y Ciencias Forenses del Ministerio Público. La determinación del perfil genético se hizo por amplificación directa de los marcadores genéticos de ADN no codificante de proteínas con el kit GlobalFiler ${ }^{\mathrm{TM}}$ Expres siguiendo las in- 
dicaciones del fabricante (Life Technologies), previamente validado. El kit utilizado incluyó 21 marcadores tipo STR autosómicos, 1 STR y 1 indel en el cromosomas Y, y amelogenina (marcador específico de sexo). Para el análisis solo se utilizaron 20 marcadores tipo STR (D8S1179, D21S11, D7S820, CSF1PO, D3S1358, TH01, D13S317, D16S539, D2S1338, D19S433, vWA, TPOX, D18S51, D5S818, FGA, D2S441, D22S1045, D10S1248, D1S1656 y D12S391) y la amelogenina no incluyendose en el estudio el STR y el indel en el cromosomas Y.

La amplificación de marcadores STR, conocida como Reacción en Cadena de la Polimerasa (PCR), se realizó empleando la técnica de amplificación múltiple, en la cual se amplificaron todos los marcadores simultáneamente. Los productos de amplificación del PCR fueron detectados mediante la técnica de electroforesis capilar con detección por fluorescencia en el Analizador Genético Applied Biosystems ${ }^{\mathrm{TM}} 3500$.

Seguidamente de la electroforesis capilar, los datos fueron importados a un software de tipificación genética, GeneMapper ${ }^{\circledR}$ ID-X v1.1, en el que se observó el perfil genético. Primero se revisaron los tamaños de los diferentes fragmentos de ADN que componen el estándar interno utilizado en cada muestra. A continuación, se revisó la asignación alélica del control positivo cuyo perfil genético se conoce previamente. Además, se revisó que el control negativo y el blanco no presentaran picos, con el fin de descartar contaminación. Los perfiles genéticos debieron ser corroborados con un marcador estándar de referencia. Para lo cual se evaluó los tamaños de los picos observados (por encima de 50 RFU), la calidad, la concentración, la presencia o no de interferencias y posibles alelos nulos.

Finalmente, luego de pasar satisfactoriamente las pruebas de control de calidad, y solo en ese caso, se procedió a exportar dichos perfiles del software GeneMapper ${ }^{\circledR}$ ID-X v1. Se realizó el cálculo de la frecuencia de un determinado perfil genético utilizando el software Familias v3.1.97 ; además se realizó el cotejo entre todos los perfiles genéticos generados y se calculó el índice de maternidad utilizando el software Familias v3.1.9.

\section{Equilibrio de Hardy Weinberg}

Para que una población se encuentre en equilibrio de Hardy-Weinberg (H-W) debe de cumplir tres requisitos: Que la población sea bastante grande, que el apareamiento ocurra producto del azar y que esta no presente ni mutaciones, ni deriva genética ni selección natural. Con ello, se predice que las frecuencias alélicas de una población permanecerán en equilibrio a través de las generaciones ${ }^{8}$. Para evaluar que los loci se encuentran en equilibrio de Hardy-Weinberg se comparó la heterocigocidad esperada (HE) y observada $(\mathrm{HO})$ según el equilibrio de Hardy-Weinberg utilizando un nivel de significación del 0,05 con el software Arlequin v3.5.2.2 en las madres y en los recién nacidos. Hernandez y Trejo" definen la HO como "la frecuencia relativa de individuos heterocigotos observada en la muestra para cualquiera de los loci" y la HE como "la probabilidad de que dos alelos tomados al azar de la población sean diferentes".

\section{Registro y almacenamiento del perfil genético}

Los perfiles genéticos generados se consideran como información personal sensible, por lo que es necesario protegerlos. Para ello se derivó una representación alfanumérica de cada uno de ellos, y se procedió a registrarlos en dos bases de datos (BD) disociadas: i) En la BD del laboratorio se grabaron el perfil genético y la representación alfanumérica indicada; ii) En la BD del RENIEC se grabó esa misma representación alfanumérica con todos los datos personales como nombre, fecha de nacimiento, dirección, etc. De esta manera la información registrada en cada BD no permitiría identificar a una persona; y sólo por mandato judicial se podrían usar las dos BD conjuntamente para obtener información personal.

La base de datos del laboratorio estaba compuesta de los perfiles genéticos vinculados con códigos alfanuméricos de 40 caracteres. Los códigos de 40 caracteres fueron una representación de los perfiles genéticos, obtenidos mediante la codificación comprimida y encriptada de la información genética, a través del algoritmo SHA 1 (Secure Hash Algorithm) ${ }^{10}$. Dicho algoritmo tuvo como característica el ser irreversible (al año 2015), es decir, no se podía obtener el perfil genético directamente a partir del código alfanumérico generado.

En la base de datos del RENIEC se encontraba el código alfanumérico de 40 caracteres vinculado a los datos personales de los recién nacidos (DNI, nombres y apellidos, fecha de nacimiento, lugar de nacimiento, datos de la madre -DNI, nombres y apellidos- y datos del padre-DNI, nombres y apellidos-) y los datos personales de las madres (DNI, nombres y apellidos, fecha de nacimiento, lugar de nacimiento).

\section{Pruebas de cotejo de identificación}

\section{Cotejos a partir de códigos alfanuméricos}

Se realizó una verificación (1:1) para corroborar que la persona es quien dice ser. Para ello, se utilizó el código alfanumérico donde se encontraban apropiadamente ligados los datos personales del recién nacido y los datos personales de la madre. Se utilizó el código alfanumérico generado por el RENIEC y se verificó a través del Registro de Identificación Genética que la persona era quien decía ser. 


\section{Relación de parentesco}

Para el presente estudio piloto, se realizó una comparación de alelos entre el recién nacido y todas las madres que participaron en el estudio (cotejo 1:n), ya que el recién nacido ha obtenido un alelo de la madre y un alelo del padre, realizándose dicha comparación para los 20 marcadores de tipo STR utilizados. Se calcularon con el Likelihood Ratio (LR) y la probabilidad de maternidad (W) en base a las frecuencias hispanas proporcionadas por el manual de Globalfiler by Life Technologies For Forensic or Paternity 2013.

\section{Likelihood Ratio (LR)}

El LR se calcula utilizando el cociente de dos probabilidades ( $\mathrm{H} 1 / \mathrm{H} 2)$ siendo $\mathrm{H} 1$ la probabilidad de obtener el genotipo del hijo suponiendo que " $\mathrm{X}$ " es la madre y $\mathrm{H} 2$ la probabilidad del genotipo del hijo suponiendo que su madre es cualquier otra mujer diferente de X (la madre asignada en la maternidad) ${ }^{11}$. Para dicho cálculos se utilizó el software Familias v3.1.97.

\section{Probabilidad de maternidad}

La probabilidad de maternidad se calculó dividiendo el valor obtenido del (LR) entre $(L R+1)$, dado que se supone que la probabilidades a priori de $\mathrm{H} 1$ y $\mathrm{H} 2$ son iguales y se expresó el resultado en porcentaje $\left((\mathrm{LR} / \mathrm{LR}+1)^{\star} 100\right)^{11}$.

\section{Consideraciones éticas}

El estudio fue aprobado por el Comité de Ética del Hospital Carlos Lanfranco La Hoz. Ambos padres firmaron previamente un consentimiento informado, el cual fue redactado siguiendo las recomendaciones de la UNESCO ${ }^{12}$. Los datos genéticos fueron irreversiblemente disociados de personas identificables. Además, la muestra biológica fue acompañada del formulario de cadena de custodia, en el que constó la individualización de todas las personas que tuvieron la muestra biológica a su cargo. Finalmente, todas las muestras biológicas fueron eliminadas, por lo que se garantiza la confidencialidad de los datos.

\section{Resultados}

Se incluyeron un total 89 individuos (44 madres y 45 recién nacidos, debido a que una de las madres tuvo gemelos, ambos del sexo masculino).

\section{Frecuencias alélicas de madres e hijos}

Las frecuencias alélicas de un marcador genético indican la cantidad de veces que se observa un alelo en la población con relación al número total de alelos de dicho marcador y se representa como una fracción o porcentaje. Entre los marcadores genéticos analizados se observó que los loci (o sistemas genéticos o marcadores genéticos) más polimórficos tuvieron 11 alelos y los menos polimórficos 5 (Tabla 1).
Se observaron alelos muy frecuentes en la población estudiada de madres e hijos para algunos marcadores genéticos como el alelo 10 del marcador D2S441 (67,00\%), alelo 15 del marcador D3S1358 (59,85\%), alelo 11 del marcador D5S818 (56,06\%), alelo 7 del marcador TH01 (54,27\%), alelo 8 del marcador TPOX (50,00\%), alelo 15 del marcador D22S1045 (46,97\%) y el alelo 14 del marcador D10S1248 (43,18\%). Al ser comparadas las frecuencias alélicas más frecuentes de la población estudiada con las frecuencias hispanas proporcionadas por el manual de Globalfiler se observa que los alelos más frecuentes de los marcadores coinciden con los identificados en presente estudio a excepción de los marcadores D12S391 (alelo 18), D13S317 (alelo 12), D16S539 (alelo 11), D18S51 (alelo 17), D21S11 (alelo 30), FGA (alelo 24) (Tabla 2).

También existen alelos raros o poco frecuentes en la población de estudio como el alelo 9 del marcador D8S1179, el alelo 27 del marcador D21S11, el alelo 11 del marcador D3S1358, el alelo 8 del marcador TH01, el alelo 8 del marcador D16S539, el alelo 16 y 21 del marcador D2S1338, el alelo 8.2 y 12.2 del marcador D19S433, el alelo 19 y 20 del marcador vWA, el alelo 10 del marcador TPOX, el alelo 11 y 19 del marcador D18S51, el alelo 14 del marcador D5S818, el alelo 18 y 28 del marcador FGA, el alelo 11.3 y 13 del marcador D2S441, el alelo 13 y 14 del marcador D22S1045, el alelo 10 del marcador D10S1248, el alelo 15.3 y 18 del marcador D1S1656, el alelo 15 y 25 del marcador D12S391. Todos los alelos mencionados están presentes en un $0,76 \%$ en la población estudiada. Al ser comparados los resultados obtenidos con las frecuencias hispanas proporcionadas por el manual de Globalfiler se observa que los alelos menos frecuentes que coinciden corresponden a los marcadores D5S818 (alelo 14), D8S1179 (alelo 9), TH01 (alelo 10) mientras que los alelos menos frecuentes del resto de marcadores no coinciden (Tabla 2).

Además, se debe de mencionar que aparecen solo en la población estudiada los alelos 8.2 del marcador D19S433, el alelo 11 del marcador D3S1358, el alelo 20.3 del marcador D12S391 y el alelo 29.2 del marcador D21S11 (Tabla 2).

\section{Equilibrio de Hardy Weinberg}

Se observó que todos los loci presentan heterocigosidad alta (mayor al $57 \%$ en los recién nacidos y en las madres). En el caso de las recién nacidos el menor valor fue de 57\% (loci D3S1358 y D5S818) y el mayor valor fue del 91\% (D2S1338) mientras que en el caso de las madres el menor valor fue el $57 \%$ (loci D3S1358 y D2S441) y el mayor valor fue del $87 \%$ (locus D18S51). Los loci analizados se encuentran en Equilibrio de Hardy Weinberg ( $p$-valor > 0,05) considerando la corrección de Bonferroni por comparaciones múltiples ${ }^{13}$. 


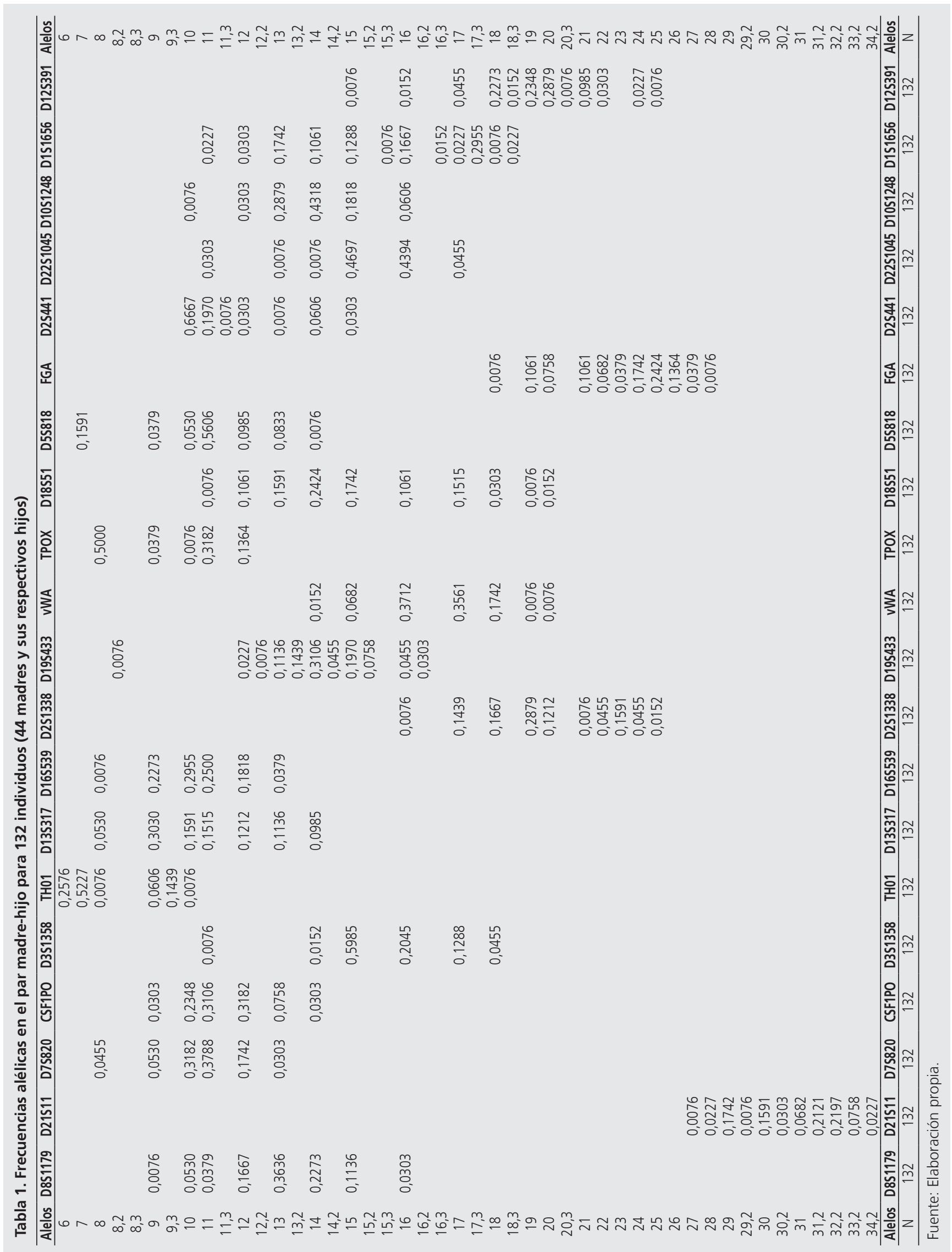




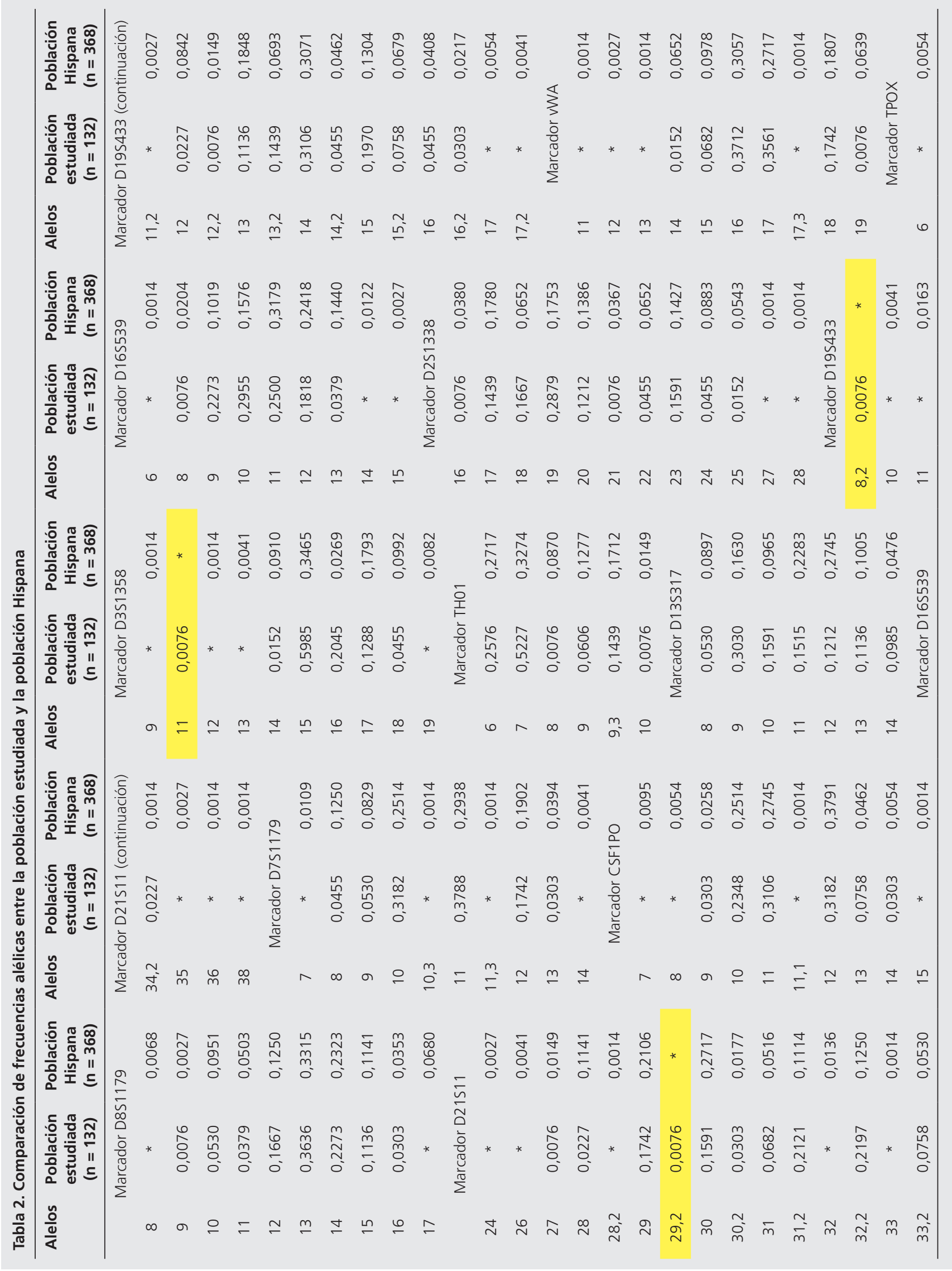




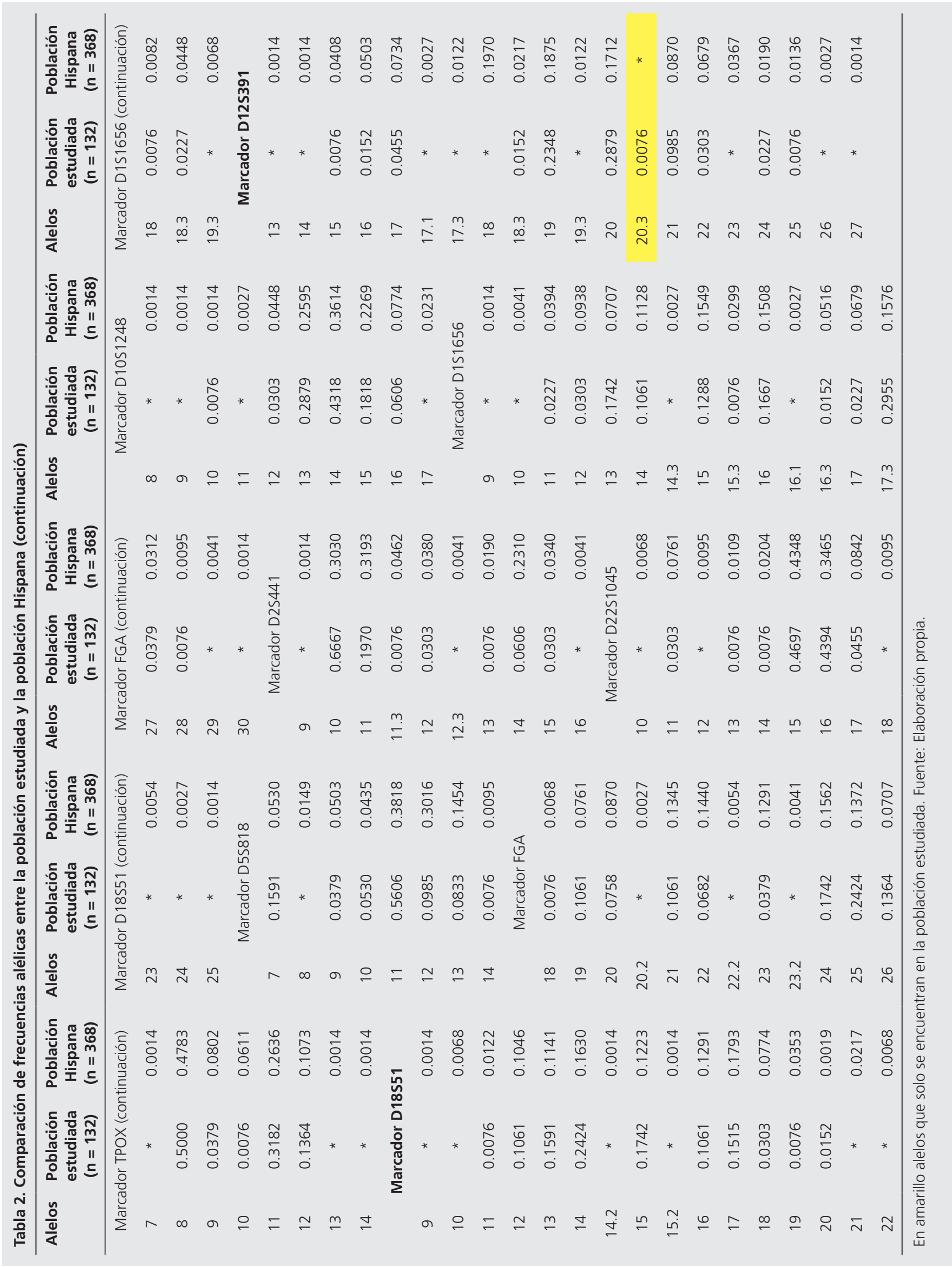




\section{Probabilidad de maternidad}

La probabilidad promedio encontrada entre 44 madres y 45 recién nacidos fue de $99.9990+0.0003$, siendo mayor de $99.9 \%$ en todos los casos (Tabla 3 ).

\section{Discusión}

El presente estudio piloto demuestra que la identificación genética de recién nacidos en un hospital público de Lima es factible ya que se aplicaron adecuadamente los protocolos y procedimientos lo que se verifica al obtener una probabilidad de maternidad mayor al $99,9 \%$ en todos los casos y lo que fue considerado como una medida de aseguramiento de la calidad. De acuerdo a Ma et al. ${ }^{14}$ la obtención de una probabilidad mayor al 99,9\% confirman la relación biológica entre dos individuos lo que está acorde con los resultados obtenidos.

Un estudio piloto similar realizado en un hospital de España muestra que la identificación genética fue factible de realizar, utilizando un protocolo fácil y rápido y materiales conocidos para los profesionales de salud. Una cantidad mínima de sangre fue suficiente para obtener el ADN para realizar las determinaciones ${ }^{15}$.

Otro estudio realizado en España comparó el método de identificación de huellas dactilares y la identificación genética del recién nacido, encontrando que ninguna huella dactilar tomada a 30 recién nacidos tuvo valor para su identificación, por el contrario, la muestra obtenida para la identificación genética del recién nacido fue suficiente para realizar las pruebas moleculares, realizando una adecuada identificación de 30 niños incluidos en el estudio ${ }^{16}$.

En el presente estudio la toma de muestra fue realizada por profesionales de la salud, la metodología empleada para la toma de sangre es ampliamente conocida por ellos, debiendo capacitarse únicamente en la obtención de la muestra de sangre en las tarjetas FTA. Así mismo, no existieron dificultades para el almacenamiento y transporte de la muestra, obteniendo material genético suficiente para su procesamiento.

Si bien no se observaron dificultades en la toma de muestra de sangre de talón en los recién nacidos, cabe resaltar que un estudio expone que la toma de muestra de sangre de cordón umbilical también podría utilizarse para la identificación genética del recién nacido, teniendo la ventaja de evitar el efecto traumático en los niños y sus posibles complicaciones como una infección. Esta evidencia podría tomarse en consideración para futuros estudio que amplíen los resultados encon$\operatorname{trados}^{17}$. En el presente estudio se utilizó la muestra de sangre de talón ya que se aprovechó la toma de muestra sanguínea realizada durante el tamizaje neonatal en el
Tabla 3. Valores de Likelihood Ratio (LR) en las muestras y probabilidad de maternidad a posteriori

\begin{tabular}{|c|c|c|}
\hline Madre/hijo & LR & Porcentaje (\%) \\
\hline 1 & 177779616.6 & 99.9999994 \\
\hline 2 & 4691189.21 & 99.9999787 \\
\hline 3 & 127294.3 & 99.9999980 \\
\hline 4 & 43746.89 & 99.9977142 \\
\hline 5 & 11168.58 & 99.9910471 \\
\hline 6 & 282673.34 & 99.9996462 \\
\hline 7 & 45313690.87 & 99.9999978 \\
\hline 8 & 691778.6 & 99.9998554 \\
\hline 9 & 1114833.63 & 99.9999103 \\
\hline 10 & 204468.03 & 99.9995109 \\
\hline 11 & 1162574990 & 99.9999999 \\
\hline 12 & 73050.25 & 99.9986311 \\
\hline 13 & 33077865.1 & 99.9999970 \\
\hline 14 & 272534640.7 & 99.9999996 \\
\hline 15 & 63479610.14 & 99.9999984 \\
\hline 16 & 41314.4 & 99.9975796 \\
\hline 17 & 52275.18 & 99.9980871 \\
\hline 18 & 1007811.55 & 99.9999008 \\
\hline 19 & 5883227.67 & 99.9999830 \\
\hline 20 & 62292760.1 & 99.9999984 \\
\hline $21 *$ & 4036084.19 & 99.9999752 \\
\hline 22 & 405617.08 & 99.9997535 \\
\hline $23^{*}$ & 4036084.19 & 99.9999752 \\
\hline 24 & 249839.08 & 99.9995997 \\
\hline 25 & 85635.09 & 99.9988323 \\
\hline 26 & 23976075.98 & 99.9999958 \\
\hline 27 & 192031.7 & 99.9994793 \\
\hline 28 & 3323408.28 & 99.9999699 \\
\hline 29 & 257853560.8 & 99.9999996 \\
\hline 30 & 110493.54 & 99.9990950 \\
\hline 31 & 32962.71 & 99.9969664 \\
\hline 32 & 105591175.9 & 99.9999991 \\
\hline 33 & 2204553.99 & 99.9999546 \\
\hline 34 & 30280589.61 & 99.9999967 \\
\hline 35 & 30731378.1 & 99.9999967 \\
\hline 36 & 167075.47 & 99.9994015 \\
\hline 37 & 210156.3 & 99.9995242 \\
\hline 38 & 2763551.13 & 99.9999638 \\
\hline 39 & 76646.65 & 99.9986953 \\
\hline 40 & 75843647.6 & 99.9999987 \\
\hline 41 & 6224.42 & 99.9839368 \\
\hline 42 & 1619646.25 & 99.9999383 \\
\hline 43 & 31952169.23 & 99.9999969 \\
\hline 44 & 72685.3 & 99.9986242 \\
\hline 45 & 1352441.92 & 99.9999261 \\
\hline
\end{tabular}

*Gemelos. Fuente: Elaboración propia. 
que obtienen sangre para realizar el tamiz de las enfermedades metabólicas y hormonales más frecuentes.

En Florida, EEUU, se recomienda la toma de muestra de sangre en las tarjetas FTA para una futura determinación de la identificación genética del recién nacido en casos de dudas. Las tarjetas con la sangre de la madre y del niño se entregan a los padres y se les recomienda que sean guardadas en un lugar seguro ${ }^{18}$.

De igual manera, la sociedad Española de Pediatría recomienda la toma de muestra de sangre de cordón umbilical, siempre con el consentimiento materno, con el único fin de comprobar la identificación del recién nacido en caso de dudas. El tiempo recomendable que puede durar estas tarjetas con el material biológico es de 1-5 años, aunque se reporta que podrían ser válidas para identificación genética hasta por 15 años ${ }^{19}$.

El almacenamiento de la muestra de sangre en tarjetas FTA resultaría adecuada en un primer momento en el Perú, con el fin de contar con el material genético correctamente almacenado, para posteriormente realizar su análisis, en caso de la implementación rutinaria de la identificación genética del recién nacido, o en caso de dudas sobre la identificación de mismo.

En cuanto a experiencias internacionales en relación a bases de datos nacionales de información genética encontramos que un registro nacional biométrico basado en ADN ya es utilizado en Kuwait ${ }^{5}$. Así mismo se está implementando una base de datos poblacional con información genética en el Emirato Árabe de Dubái, el cual será utilizado principalmente para la prevención y tratamiento de enfermedades ${ }^{20}$. En China existe una base de datos nacional de ADN que contiene datos padres de niños perdidos, niños víctima de trata, extraviados o sin hogar. Un estudio reciente basado en este registro, recomienda el uso de más de 18 marcadores genéticos para una correcta identificación de padres e hijos ${ }^{21}$. A nivel de Perú, este es el primer estudio piloto que evalúa la factibilidad de esta tecnología para fines de identificación.

En el presente estudio, se identificaron etapas del proceso de identificación genética que podrían mejorarse para una futura implementación de esta tecnología a una mayor escala en el Perú, como, por ejemplo, la automatización para la obtención, análisis y encriptación de los perfiles genéticos. La toma de muestra fue fácilmente aplicada por personal de salud, sin embargo, la recolección y el almacenamiento de la misma, implica una actividad adicional en la atención del recién nacido por parte de la enfermera. En este estudio, la recolección y el almacenamiento de estas tarjetas con la sangre del niño, estuvo a cargo de un personal del RENIEC, contratado específicamente para este proceso. Es por tanto necesario una fuerte colaboración institucional entre el MINSA y el RENIEC para poder replicar esta experiencia en otros hospitales del Perú.
Dentro de las limitaciones del presente estudio se encuentra el poco número de muestras, y la aplicación en un solo hospital totalmente accesible desde el centro de la ciudad. Sin embargo, este hospital tiene las mismas dificultades logísticas y administrativas de otros hospitales de Lima y las regiones del Perú, lo cual podría dar una idea de la factibilidad de esta técnica en hospitales del mismo o mayor nivel resolutivo. Otra limitación es que muchos nacimientos a nivel nacional, se dan en establecimientos del primer nivel de atención (centros de Salud), en los cuales el número de personal y recursos son mucho menores que en Hospitales, por lo que se tendría que explorar la viabilidad de la toma de muestra y el transporte desde comunidades alejadas hasta la institución que realizará la identificación genética.

En conclusión, la identificación genética fue factible de realizar de manera conjunta entre el RENIEC, un hospital y el laboratorio (LABIMOG). El RENIEC gestionó adecuadamente todo el proceso incluido el registro de consentimiento, el cargo de envío de muestras biológicas y el registro de Identificación genética. El protocolo de toma de muestra fue fácilmente aplicado por personal de salud capacitado, el almacenamiento y transporte del material biológico se realizó sin dificultades hasta la institución donde se realizó la determinación de la identificación genética. El LABIMOG realizó de manera adecuada el procesamiento de la muestra y la obtención del perfil genético. Finalmente, todo ello se ve reflejado en la probabilidad de maternidad cuyo valor en todos los caso fue superior al 99,9\%.

Estos resultados podrían servir para la posible implementación de esta técnica, con el fin de realizar la identificación inequívoca del recién nacido en caso de sospecha o dudas referente a la identificación, sin embargo, es necesario realizar un estudio de evaluación de esta tecnología sanitaria (ETS) en el contexto peruano, así como de otros estudios piloto para recomendar su implementación a mayor escala para identificación por perfiles genéticos en personas naturales para mejorar el proceso de identificación de menores de edad, apoyar al sistema jurídico, identificación segura en caso de tratas de personas, colaborar en la solución de problemas de paternidad (cuando sea requerido legalmente) $\mathrm{y}$ apoyo al sistema forense.

Futuros estudios deberían evaluar la factibilidad de otros tipos de toma de muestra biológica, porque pueden existir casos en los que no se autorice la toma de muestra sanguínea en tarjetas FTA, y el estudio de otras tecnologías de obtención de perfiles genéticos, así mismo incluir establecimientos de salud del primer nivel de atención, procedentes de sierra y selva peruana, con el fin de evaluar las dificultades existentes para la aplicación de esta técnica a nivel nacional. 


\section{Responsabilidades Éticas}

Protección de personas y animales: Los autores declaran que los procedimientos seguidos se conformaron a las normas éticas del comité de experimentación humana responsable y de acuerdo con la Asociación Médica Mundial y la Declaración de Helsinki.

Confidencialidad de los datos: Los autores declaran que han seguido los protocolos de su centro de trabajo sobre la publicación de datos de pacientes.

Derecho a la privacidad y consentimiento informado: Los autores han obtenido el consentimiento informado de los pacientes y/o sujetos referidos en el artículo. Este documento obra en poder del autor de correspondencia.

\section{Conflicto de intereses}

Los autores declaran no tener conflicto de intereses.

\section{Financiamiento}

Registro Nacional de Identificación y Estado Civil (RENIEC) Perú.

\section{Agradecimientos}

\begin{abstract}
Se agradece a la Gerencia de Calidad e Innovación y a la Gerencia de Tecnología de la Información del RENIEC por el apoyo invaluable durante toda la realización del estudio piloto. Al Hospital Carlos Lanfranco La Hoz de Puente Piedra (Lima-Perú) por el apoyo durante la toma de muestra a las personas participantes en el estudio piloto y al Laboratorio de Biología Molecular y de Genética del Instituto de Medicina Legal y Ciencias Forenses del Ministerio Público por el procesamiento y obtención de los perfiles genéticos que fueron uno de los insumos más importantes para realizar la validación de nuestros protocolos y procesos.
\end{abstract}

\section{Referencias}

1. Constitución Política del Perú. 1993

2. Convención sobre los Derechos del Niño UNICEF. 1989. Disponible en: http:// www.un.org/es/events/childrenday/pdf/ derechos.pdf

3. Kotzerke J, Davis SA, Hayes R, Horadam KJ. Newborn and infant discrimination: revisiting footprints. Aust J Forensic Sci. 2017;1-14.

4. Champod C, Tistarelli M. Biometric Technologies for Forensic Science and Policing: State of the Art. In: Tistarelli M., Champod C. (eds) Handbook of Biometrics for Forensic Science. Advances in Computer Vision and Pattern Recognition. Springer, Cham. 2017.

5. Santurtún A, Lema C, Zarrabeitia MT. Derechos fundamentales en el contexto de las bases de datos forenses: Revisión y análisis de la Ley 78/2015 de Kuwait. Rev Esp Med Leg. 2017;43:79-86.

6. GE Healthcare Life Sciences. Whatman FTA Brochure; Your forensic samples, our experience. 2011 [citado el 9 de noviembre de 2015]; Disponible en: https://www.thermofisher.co.nz/Uploads/ file/Supplier-Partners/GE-Whatman-FTA. pdf.

7. Kling D, Tillmar AO, Egeland T. Familias 3-Extensions and new functionality. Forensic Sci Int: Genetics 2014;13:121-7.
8. Filho Rigotti J. Comparación de la frecuencia alélica de 13 loci STRs de la población brasileña y española para fines de identificación humana en genética forense. [Tesis doctoral]. Valladolid: Universidad de Valladolid; 2012.

9. Hernández AW, Trejo F. Estudio Genético Poblacional de Frecuencias Alélicas para 15 marcadores STR presentes en la Población del Estado de Zacatecas Aplicado a la Práctica Forense. Archivos de Medicina 2014;10:1-24.

10. Shah CS, Panchal DR. Secured Hash Algorithm-1: Review Paper. Int J Adv Res Eng Tech 2014;2:26-30.

11. Prieto, L. Valoración de la prueba de ADN: Métodos básicos. En: Curso "Familial testing and mixtures". España: Universidad Santiago de Compostela; 2015. Disponible en: http://familias. name/01-Metodos-basicos.pdf.

12. Organización de las Naciones Unidas para la Educación, la Ciencia y la Cultura. Declaración Internacional sobre los Datos Genéticos Humanos: [Internet]. UNESCO; 2003. Portal.unesco.org. 2017 [citado el 9 de mayo de 2017]. Disponible en: http://portal.unesco.org/es/ev.phpURL_ID $=17720 \& U R L \_D O=D O$ TOPIC\&URL_SECTION $=201 . \mathrm{html}$.

13. Bonferroni, CE. Teoria statistica delle classi e calcolo delle probabilità. Pubblicazioni del R Istituto Superiore di Scienze Economiche e Commerciali di
Firenze. 1936;8:3-62.

14. Ma H, Zhu H, Guan F, Cherng S. Paternity Test. J Am Sci. 2006;2:76-92.

15. Lorente MJ, Lorente JA, Lorente M, et al. Genetic maternal-child identification with DNA. Rev Enferm 1999;22:547-52.

16. Sanz MC, Espinal MI, Domínguez A, Fernández S, Cardesa F, Hernández M. La identificación del recién nacido: Asignatura pendiente. Matronas Prof0 2012;13:66-72.

17. Crouch SJ, Rowell KR, Beiser SO. Umbilical cord blood for newborn DNA identification. J Obstet Gynecol Neonatal Nurs. 2007;36:308-312.

18. McCartney P. Newborn Identification, Screening, and DNA. MCN Am J Matern Child Nurs. 2003;28:124.

19. Sanz E, Sánchez M, Rite S, Benavente I, Leante J, Pérez A, Ruiz C, Sánchez M. Recomendaciones para la identificación inequívoca del recién nacido. An Pediatr 2017;87:235.

20. Dankar FK, Ptitsyn A, Dankar SK. The development of large-scale de-identified biomedical databases in the age of genomics-principles and challenges. Hum Genomics. 2018;12(1):19.

21. Yu K., Fung WK. Evaluation of parentage testing accuracy of child trafficking cases: Combining the exclusion probability and likelihood ratio approaches. Forensic Science International: Genetics. 2018;34:81-7. 